Meta

Journal des traducteurs

Translators' Journal

\title{
Bilinguisme officiel et traduction au Canada : les interprétations littéraires de Patrice Desbiens et de Jacques Brault / E. D. Blodgett
}

\section{Catherine Leclerc}

Volume 59, numéro 3, décembre 2014

Traduction et plurilinguisme officiel

Translation and Official Multilingualism

URI : https://id.erudit.org/iderudit/1028654ar

DOI : https://doi.org/10.7202/1028654ar

Aller au sommaire du numéro

Éditeur(s)

Les Presses de l’Université de Montréal

ISSN

0026-0452 (imprimé)

1492-1421 (numérique)

Découvrir la revue

Citer cet article

Leclerc, C. (2014). Bilinguisme officiel et traduction au Canada : les interprétations littéraires de Patrice Desbiens et de Jacques Brault /

E. D. Blodgett. Meta, 59(3), 494-516. https://doi.org/10.7202/1028654ar
Résumé de l'article

Transfiguration de Jacques Brault et E. D. Blodgett (1998), et L'homme invisible/The Invisible Man (1981) de Patrice Desbiens se situent à la croisée des langues officielles canadiennes. L'un est un échange entre un poète québécois et un poète albertain ; l'autre, le récit de l'expérience entre les langues d'un protagoniste franco-ontarien. Ces textes poétiques empruntent - mais pour les détourner - les codes de l'édition bilingue. Ils ont tous deux été lus comme des parodies du bilinguisme symétrique promu par la Loi sur les langues officielles du Canada. Le présent article veut rendre compte de leur relation, à la fois idéologique et formelle, avec le bilinguisme officiel et avec les pratiques traductionnelles qui lui sont associées. Il s'intéresse autant au cadre commun que le bilinguisme officiel leur procure qu'aux manières, divergentes, dont il s'en démarque. En ce sens, il propose un comparatisme portant non seulement sur les imaginaires respectifs de l'anglais et du français au Canada et sur leurs zones de contact, mais surtout sur les enjeux des différents types de rapports à la traduction émanant de telles zones. D’une tension entre deux espaces sociolinguistiques à la fois séparés et en interaction, il invite à déplacer le regard vers l'hétérogénéité de leurs lieux de rencontre les plus intenses.
Ce document est protégé par la loi sur le droit d'auteur. L'utilisation des services d’Érudit (y compris la reproduction) est assujettie à sa politique d'utilisation que vous pouvez consulter en ligne.

https://apropos.erudit.org/fr/usagers/politique-dutilisation/ 


\title{
Bilinguisme officiel et traduction au Canada: les interprétations littéraires de Patrice Desbiens et de Jacques Brault / E. D. Blodgett
}

\author{
CATHERINE LECLERC \\ Université McGill, Montréal, Québec \\ catherine.leclerc@mcgill.ca
}

\section{RÉSUMÉ}

Transfiguration de Jacques Brault et E. D. Blodgett (1998), et L'homme invisible/The Invisible Man (1981) de Patrice Desbiens se situent à la croisée des langues officielles canadiennes. L'un est un échange entre un poète québécois et un poète albertain; l'autre, le récit de l'expérience entre les langues d'un protagoniste franco-ontarien. Ces textes poétiques empruntent - mais pour les détourner - les codes de l'édition bilingue. Ils ont tous deux été lus comme des parodies du bilinguisme symétrique promu par la Loi sur les langues officielles du Canada. Le présent article veut rendre compte de leur relation, à la fois idéologique et formelle, avec le bilinguisme officiel et avec les pratiques traductionnelles qui lui sont associées. II s'intéresse autant au cadre commun que le bilinguisme officiel leur procure qu'aux manières, divergentes, dont il s'en démarque. En ce sens, il propose un comparatisme portant non seulement sur les imaginaires respectifs de l'anglais et du français au Canada et sur leurs zones de contact, mais surtout sur les enjeux des différents types de rapports à la traduction émanant de telles zones. D'une tension entre deux espaces sociolinguistiques à la fois séparés et en interaction, il invite à déplacer le regard vers l'hétérogénéité de leurs lieux de rencontre les plus intenses.

\begin{abstract}
Transfiguration by Jacques Brault and E. D. Blodgett (1998), and L'homme invisible/The Invisible Man by Patrice Desbiens (1981) are located at the crossroad of Canada's official languages. The first is an exchange between a Québécois and an Albertan poet; the second narrates the bilingual experience of a Franco-Ontarian protagonist. Both of these texts have been commented upon for their parodies of the symmetrical bilingualism promoted by Canada's Official Languages Act. This article describes their ideological and formal relationships with official bilingualism and with the translation practices associated with it. It focuses on the common framework official bilingualism grants them and on the various strategies explored by the authors to subvert this framework. The texts studied show two very different reactions that put translation to work in contrasting ways. As a result, this article's conclusion calls for a comparatism that, instead of limiting its exploration to the differences between English and French or even their contact zone, concentrates on the different relationships with translation emanating from that very zone. In the narrow interstice between English and French lies a world as heterogeneous as the two sociolinguitic spaces it both joins and opposes.
\end{abstract}

\section{MOTS CLÉS/KEYWORDS}

bilinguisme officiel au Canada, littérature bilingue, E. D. Blodgett, Jacques Brault, Patrice Desbiens

official bilingualism in Canada, literary bilingualism, E. D. Blodgett, Jacques Brault, Patrice Desbiens 


\section{Bilinguisme officiel, politiques et poétiques traductionnelles}

Au Canada, la charge idéologique entourant le bilinguisme anglais-français est immense. Encadré par une politique officielle qui le valorise en affirmant l'égalité des deux langues, ce bilinguisme fait en même temps l'objet de méfiance, certains le considérant comme superflu et d'autres jugeant insuffisante la protection qu'il accorde au français, langue minoritaire. C'est que l'égalité de statut que la loi confère aux deux langues masque - en même temps qu'elle y répond - leur rapport asymétrique, mouvementé. D'une part, la correction partielle que la loi apporte à une inégalité issue tant de la démographie que de l'histoire coloniale semble nier cette inégalité (par exemple dans l'adéquation des minorités de langue officielle l'une avec l'autre), qui n'en perdure pas moins dans d'autres sphères de la vie publique. De l'autre, officialisant l'importance symbolique du français, elle accorde à celui-ci un avantage qui tend à délégitimer les tentatives de protection des minorités francophones.

Les résultats de cette situation se font sentir jusque sur la scène littéraire. Ainsi, l'anthologie de récits et d'entrevues Other Solitudes: Canadian Multicultural Fiction, publiée en 1990, se situe dans le sillage de la réponse qu'offre le multiculturalisme canadien - autre politique gouvernementale - au bilinguisme officiel. Dans la préface et l'introduction, les coéditrices, Linda Hutcheon et Marion Richmond, affirment vouloir contester par cet ouvrage l'habituelle «hierarchy of social and cultural privilege» qu'on trouve au pays (Hutcheon 1990: 2). Dans la même foulée, elles opposent les écrits de leur anthologie à ceux des «two official cultures» (Richmond 1990: ix), qu'elles présentent comme traditionnellement dominantes (voir Hutcheon 1990: 2) sans faire de véritable distinction entre elles. La reconnaissance officielle qui les caractérise, ici, justifie le présupposé de symétrie.

L'équivoque est encore plus apparente en ce qui concerne les politiques de traduction associées au bilinguisme officiel. En effet, suivant la loi, les documents officiels produits par l'État fédéral canadien doivent l'être aussi bien en français qu'en anglais. Conformément à l'égalité de statut des deux langues, les versions anglaise et française ont en outre toutes deux valeur d'original. En découle un travail de traduction continuel de la part de l'État, travail qui assure la symétrie de la production langagière gouvernementale. Ce travail est par ailleurs redoublé dans d'autres domaines de la vie canadienne. Par exemple, les étiquettes des produits de consommation sont elles aussi assujetties à une exigence de bilinguisme. Or, comme la création de tels documents s'effectue le plus souvent dans la langue de la majorité, c'est de l'anglais vers le français qu'ont lieu la plupart des traductions (voir Blodgett 1982: 29-30; Juel 1999: 239; Taravella 2011: 4).

Dans ce contexte, la traduction acquiert à la fois une importance considérable et des connotations négatives. D'un côté, les coûts qui y sont associés inquiètent certains contribuables et groupes de pression (Reid 1993; Vaillancourt et Coche 2009; Vaillancourt, Coche et al. 2012). Bien que la manière dont ces coûts sont estimés ne fasse pas consensus, l'insistance sur leur importance, reprise dans les médias, nourrit l'impression «que les anglophones paient un excès de taxes pour que les francophones puissent jouir de services inutiles», ainsi que l'ironise Edmund A. Aunger (2012: 6). D'un autre côté, le fait que de nombreux éléments discursifs de leur vie quotidienne ne soient accessibles aux francophones que par des formulations créées à partir d'une autre langue préoccupe tant les experts que les locuteurs du français 
eux-mêmes et contribue à une perception de la traduction comme menace. Dans son analyse du «regard doxologique sur la traduction" à travers l'histoire du Canada français, Sherry Simon fait remarquer, à l'aide de nombreux exemples, que « [c]e sont souvent les effets délétères de la traduction sur la langue qui ont été relevés» (voir 1994: 37; 39-40).

Quelles que soient les positions des uns et des autres à son endroit, le bilinguisme officiel, dans son versant traductionnel, imprègne l'imaginaire national canadien. D’une part, officialisant le statut des minorités de langue officielle partout au pays, il en est venu à leur être étroitement associé: la connaissance et l'usage des deux langues officielles sont en effet devenus des caractéristiques définitoires de ces minorités, surtout dans le cas des francophones. ${ }^{1}$. D'autre part, même chez les anglophones et même chez ceux parmi eux (nombreux) qui ne maîtrisent pas le français, le bilinguisme anglais-français joue un rôle non négligeable dans la construction de l'identité nationale. En témoigne la campagne publicitaire d'une compagnie de bière, au début des années 2000, dont le slogan était «I am Canadian». Cette campagne a atteint une popularité record grâce à un monologue nationaliste dont le protagoniste affirmait fièrement: "I speak English and French.» Rien dans son discours - que Molson, la compagnie en question, ne fit jamais circuler en traduction française - ne permettait de conclure que ce protagoniste était effectivement en mesure de converser en français; par contre, la mise en valeur du français comme composante de son identité lui permettait de se distinguer des Américains, et tel était le but explicite de cette publicité. Les détracteurs du bilinguisme officiel reconnaissent d'ailleurs euxmêmes, statistiques à l'appui, l'attachement d'une majorité de la population canadienne à cette politique; sans compter que c'est au Québec, où les critiques ont été les plus vives, que cet attachement est le plus grand (voir par exemple Vaillancourt et Coche 2009: 9).

Les textes littéraires sur lesquels le présent article se penche sont deux œuvres aux poétiques résolument traductionnelles, qui prennent pour point de départ la symétrie caractéristique de la traduction de documents officiels entre l'anglais et le français au Canada et qui exploitent son potentiel de symbolisation. Adoptant à cette fin une forme qui les rapproche singulièrement, tous deux empruntent - mais pour les détourner - les codes de l'édition bilingue. Ainsi, L'homme invisible/The Invisible Man du poète franco-ontarien Patrice Desbiens est paru en 1981 aux Éditions Prise de parole, en association avec Penumbra Press, un éditeur de langue anglaise de la même région au nord de l'Ontario. Dix-sept ans plus tard, en 1998, paraissait Transfiguration, coécrit par le Québécois Jacques Brault et l'Albertain E. D. Blodgett. Le recueil était une collaboration entre les Éditions du Noroît au Québec et les éditions BuschekBooks, sises à Ottawa. Outre un intérêt pour la poésie, que les deux éditeurs ont en partage et sur laquelle le Noroît se concentre exclusivement, BuschekBooks a pour mandat de publier des ouvres en traduction, et notamment en édition bilingue. La formule de l'édition bilingue subit toutefois, dans les deux cas étudiés ici, d'importants réaménagements.

Dans Transfiguration (voir annexe 1), Blodgett et Brault créent tour à tour, chacun dans sa langue maternelle (l'anglais pour Blodgett et le français pour Brault), un poème qui sert d'inspiration au poème suivant, écrit dans l'autre langue par l'autre poète, et figurant sur la page suivante. L'ordre de parution des textes, avec ceux de Blodgett (à qui revient l'initiative du projet) en ouverture sur la page de gauche, donne 
l'impression d'une traduction de l'anglais vers le français. C'est d'ailleurs Jacques Brault seul qui, pour ce recueil, remportait en 1999 le prix de traduction de l'anglais au français du Gouverneur général du Canada, Transfiguration étant ainsi ramenée aux catégories préconstruites d'œuvre originale (anglaise) et de traduction (française). Pourtant, sur les pages bilingues produites par Blodgett et Brault, chaque poème «original» est à la fois texte source (il est générateur du poème suivant) et texte cible (il est une réponse au poème précédent). À ces textes de départ s'ajoutent, au bas de chaque page, des traductions plus conventionnelles, la trame du bas redonnant dans l'autre langue le poème de la trame du haut. Sans doute est-ce à propos de ces traductions que le jury des Prix littéraires du Gouverneur général louait la «virtuosité» et la «précis[ion]» de la langue de Brault ${ }^{2}$. Dans les traductions de la trame du bas, l'ordre d'apparition des langues s'inverse par rapport à la trame du haut. La double page à quatre textes peut dès lors se lire dans tous les sens; elle autorise plusieurs permutations (voir C. Melançon 2000: 12). Ces multiples trajectoires de lecture possibles font écho à la démarche des auteurs, que Brault qualifie dans son texte liminaire «d'écriture oblique du poème», faisant signe à son vis-à-vis (Brault in Brault et Blodgett 1998: 9).

Dans L'homme invisible/The Invisible Man (voir annexe 2), Patrice Desbiens occupe à lui seul tout l'espace de la page double. Chez lui, c'est le français qui est à gauche et l'anglais, à droite. À première vue, avec un seul texte par page, il maintient davantage que Blodgett et Brault la division des langues entre la page de gauche et celle de droite. Pourtant, son texte s'écarte des conventions propres aux éditions bilingues encore plus intensément que celui de Blodgett et Brault. Des irruptions sur la page française de la langue anglaise - langue qui, dans une édition bilingue conventionnelle, devrait être celle de la traduction - perturbent cette division des langues, surtout qu'elles se chargent de signification. De plus, Desbiens use de maintes autres stratégies pour renverser la direction attendue de la traduction, c'est-à-dire de la langue et du texte sources vers la langue et le texte cibles. Mais la perturbation la plus importante a lieu ailleurs: bien qu'il y ait redondance partielle entre les versions anglaise et française du texte, les deux ne se redoublent pas entièrement, même sur le plan diégétique. Sur ce point, Desbiens déjouait avec L'homme invisible/The Invisible Man jusqu'aux attentes de son éditeur, dont le projet était de permettre aux anglophones, interlocuteurs quotidiens des Franco-Ontariens, d'avoir accès dans leur langue à l'œuvre du poète (voir Tremblay 1996: 206-207). Or, on a plutôt affaire ici à un récit en deux langues, où la duplication offerte par la traduction (gage des éditions bilingues) est à la fois incomplète et inconstante. La lecture des deux pages est nécessaire à l'appréhension du texte dans sa globalité. En fait, vu l'unité du récit bilingue, on pourrait même aller jusqu'à avancer qu'il n'y a pas deux langues dans L'homme invisible/The Invisible Man, mais bien une seule, coincée entre deux versions sans commune mesure.

Le détournement de la formule de l'édition bilingue auquel s'adonnent Desbiens d'un côté et Brault et Blodgett de l'autre a reçu d'emblée, dans les deux cas, une interprétation critique en lien, mais aussi en contraste, avec les politiques canadiennes sur les langues officielles. De fait, dans leur manipulation de la formule de l'édition bilingue, Transfiguration et L'homme invisible/The Invisible Man entretiennent un dialogue idéologique et formel avec le bilinguisme officiel canadien et avec les pratiques de traduction qui lui sont associées. C'est ce dialogue que le présent article examinera. 


\section{Transfiguration, ou le bilinguisme officiel revu, corrigé, transfiguré}

Le lien avec le bilinguisme officiel n'est pas explicite dans le texte de Blodgett et Brault. Au contraire, Transfiguration semble se caractériser par un éloignement de tout enjeu politique. L'inspiration que les poètes se reconnaissent - et à laquelle Blodgett assimile explicitement leur démarche dès l'incipit du recueil (voir Blodgett in Blodgett et Brault 1998: 8) - est le renga, un art poétique japonais dont la popularité a culminé il y a plusieurs siècles (voir Horton 1993: 443; Brazell et Cook in Konishi 1975: 29). Pour le dire dans les termes de Charlotte Melançon, Transfiguration appartient donc «à un genre culturel étranger» (2000: 11), dans le temps comme dans l'espace. Un renga est un poème écrit en collaboration. Il est formé de strophes composées en alternance par ses participants et s'enchaînant les unes aux autres suivant certaines règles. Ces règles complexes, Blodgett et Brault ne s'y astreignent pas systématiquement: Brault fait valoir d'entrée de jeu que les poètes suivent ici chacun «sa dictée propre» et qu'ils ne se sont donné «aucune règle préétablie» (Brault in Blodgett et Brault 1998:9). Néanmoins, plusieurs des composantes de l'univers de Transfiguration - à distance de la vie en société, et à fortiori des questions d'actualité sur le contact des langues officielles au Canada - peuvent être rattachées aux règles du renga. Que le recueil soit avant tout descriptif, qu'il dépeigne le passage des saisons, que son lexique renvoie à la nature (et qu'il y soit question en particulier d'oiseaux ${ }^{3}$, de fleurs et de la lune), qu'il célèbre l'amitié et enfin que ces éléments s'entremêlent avec une grande unité de ton sont autant de traits qui relèvent de ces règles (voir Konishi 1975). Passer par le renga permet donc à Blodgett et Brault de se rejoindre en terrain neutre et harmonieux. Traditionnellement - et nos poètes répondent à ces critères -, les participants à la création d'un renga sont un groupe d'individus « who share a degree of mutual friendship as well as similar levels of competence» (Konishi 1975: 33). En outre, bien que le renga ne soit pas plurilingue par tradition, un exemple occidental célèbre de renga plurilingue (en anglais, espagnol, français et italien), publié chez Gallimard en 1971, sert de précédent à Transfiguration: Renga d'Octavio Paz, Jacques Roubaud, Edoardo Sanguineti et Charles Tomlinson.

Malgré l'établissement d'un paradigme étranger et malgré l'absence de thématisation, la juxtaposition symétrique de l'anglais et du français que propose le recueil semble avoir imposé une analogie avec le bilinguisme officiel canadien. Sherry Simon, à tout le moins, qui a le plus abondamment commenté Transfiguration, établit précautionneusement une telle analogie: "Though Transfiguration avoids explicit political references, it could perhaps be read as a parody of symmetrical bilingualism » (2006: 140). Dans la pensée de Simon, l'opposition entre «the pervasive, neat, matching paragraphs of official bilingualism » et «the irregular shape of translation » (2002: 22) de textes littéraires qui «baffle and upset official images of symmetrical dialogue» (1999: 60) est récurrente. Mais cette opposition trouve aussi à s'alimenter dans les écrits antérieurs des deux poètes, eux-mêmes chercheurs et essayistes.

À l'encontre du discours de méfiance dont il était alors entouré, Jacques Brault a été un des premiers intellectuels franco-québécois à connoter positivement, dès ses Poèmes des quatre côtés en 1975, la traduction de l'anglais (voir Simon 1994: 59; Larose 2004: 352 et 369-370; Côté 2007: 164), à laquelle il s'adonnait à cette occasion. En 1977, dans «Sur la traduction de la poésie», il réitérait sa conviction du potentiel de la traduction comme «reculturation vivifiante» et "véritable odyssée désalié- 
nante» pour les poètes francophones du Québec (voir Brault 1989: 213). S’appuyant sur la démarche de traduction qu'il avait adoptée dans Poèmes des quatre côtés, il affirmait: «La langue anglo-américaine m'agressait? Eh bien! je traverserais cette langue, je la traverserais jusqu'à ma langue propre (et inconnue), et au cours de cette traversée pénible et salutaire, je me perdrais dans l'autre et l'autre se retrouverait en moi» (1989: 212)4. À l'accomplissement de ce potentiel, il émettait toutefois une importante condition: "Pour arriver à cette circulation des poèmes québécois dans le monde et des poèmes étrangers au Québec, il faut cependant dissiper la croyance fumeuse au biculturalisme institutionnalisé, monstre politique qui trimbale, attachée à sa queue, la casserole du bilinguisme officiel» (1989: 213). Quant à Blodgett, c'est bien lui qui affirmait, en introduction à son recueil d'études comparatistes intitulé Configuration. Essays in the Canadian Literatures: "binarism in Canada, while it is a violent stasis, masks, in fact, an anglophone hegemony» (1982: 9). Plus loin dans cet ouvrage, empruntant le terme à Tony Wilden, il mettait son lectorat en garde contre les démarches de «symétrisation», qui nient la réalité des rapports de pouvoir entre les groupes en cause et dès lors consolident l'ascendant de la langue dominante. Blodgett ajoutait: "any literary framework that assumes equality of status between these two cultural groups [Blodgett les désigne comme étant "canadian-québécois»] mistakes the nature of the relationship» (1982: 32-33). Pour Blodgett comme pour Brault, l'asymétrie entre l'anglais et le français est un donné social qu'il ne sert à rien de nier, et à partir duquel il faut au contraire travailler pour un tant soit peu le contrer.

Avec Transfiguration, les deux poètes créent précisément l'univers d'échanges réciproques dont ils affirment qu'il est difficilement envisageable dans l'espace sociopolitique où la Loi sur les langues officielles est appliquée. Ce faisant, ils corrigent la loi, la réécrivent. Dans le nouvel horizon où ils choisissent de se situer, celui du renga, chaque poème de l'un devient une offrande faite à l'autre. Contre la logique de la loi, c'est cette logique de l'offrande qui est mise de l'avant dans le texte, comme en témoigne ce passage de Blodgett:

(1) are we agreed on this my friend the moon's a word I give to you (Blodgett in Blodgett et Brault 1998: 40)5.

La logique de l'offrande permet aux auteurs de faire - telle sera la réponse de Brault - "amitié avec des mots» (Brault in Blodgett et Brault 1998: 41). Ces mots, Brault leur procure une adéquation qui garantit le rapprochement avec son interlocuteur, puisqu'il ajoute à leur sujet: "où le son est le sens » (41). Le passage, qui met aussitôt l'affirmation en acte, se lit comme suit:

(2) faisant amitié avec des mots où le son est le sens la mésange à capuchon noir pousse du bec son bref fi-bi [...] (Brault in Blodgett et Brault 1998: 41)

sealing friendship with words that mean what you hear the chickadee with its black cap eases from its beak its soft phee-bee [...] (Brault tr. Blodgett in Blodgett et Brault 1998: 41) 
On reviendra plus loin sur l'adoucissement que Blodgett fait subir aux images de Brault - manifeste ici lorsque «pousse» devient «eases» et «bref» devient «soft». Pour l'instant, notons que les onomatopées, à la fois semblables et différentes («fi-bi» et "phee-bee»), matérialisent la correspondance mêlée d'écart entre les deux poètes. Brault résume cette double position dans son texte liminaire:

(3) Ainsi avons-nous écrit en étrange familiarité, grâce à une amitié qui ne s'est pas donné d'alibi en cherchant à gommer nos différences. (Brault in Blodgett et Brault 1998: 9)

C’est précisément cette jonction entre différence et familiarité que l'onomatopée met en acte. D’une part, elle place les poètes en terrain presque commun, le mimétisme entre son et sens tenant lieu d'universel; de l'autre, la phonétique et l'orthographe différentes $\mathrm{du}$ français et de l'anglais maintiennent une distance, elles introduisent une variation qui rend le même autre. La différence est à la fois infime et éminemment repérable.

Par delà des thèmes et expressions qui facilitent l'entente, toute la structure du recueil est modelée de façon à adoucir les heurts. Tandis que chaque poème "original» rend hommage au précédent en le poursuivant, les traductions, elles (où Brault reprend dans sa langue le propos de Blodgett et vice versa), aménagent des zones de transition entre les écrits de chaque poète. Ainsi, le «but time/made flesh» de Blodgett, traduit par Brault par «mais le temps/fait chair» (Blodgett et Brault 1998: 82), devient dans le poème suivant de Brault «c'est encore elle la chair du temps » (Brault in Blodgett et Brault 1998: 83), avant de redevenir flesh dans la traduction de Blodgett: «there it is again the flesh of time» (Blodgett in Blodgett et Brault: 83), sans qu'on sache avec certitude où les voix des poètes se relaient. Ailleurs, l'usage des pronoms désignant les oiseaux s'harmonise. Pour la plupart de ses mentions d'oiseaux, Blodgett emploie tel qu'attendu le pronom neutre «it». Il le fait dans sa traduction des oiseaux de Brault:

(4) burn as the lark burns high upon its extasy (Brault tr. Blodgett in Blodgett et Brault 1998: 11)

(5) a crow in its brilliant body (Brault tr. Blodgett in Blodgett et Brault 1998: 13)

Il le fait également dans ses propres poèmes:

(6) a sparrow sits upon a fence [...] if it sang its song would never reach the ground (Blodgett in Blodgett et Brault 1998: 48)

Il conserve cette stratégie même lorsque Brault, par une pirouette syntaxique, féminise un oiseau masculin:

(7) [...] elle prend la tangente cette folie de geai bleu (Brault in Bodgett et Brault 1998: 15)

\section{[...] it moves} obliquely

this blue jay madness (Brault tr. Blodgett in Blodgett et Brault 1998: 15)

Mais il arrive aussi à Blodgett - dont le choix s'appuie sans doute sur la polysémie du terme «hairy» en anglais - de reprendre le genre que Brault, suivant les règles du français, assigne à son oiseau: 
(8) le pic chevelu

que signe à la tête une étoile de sang (Brault in Blodgett et Brault 1998: 47, je souligne; voir aussi 67)

figured on his head

the hairy woodpecker

a star of blood (Brault tr. Blodgett in Blodgett et Brault 1997: 47, je souligne; voir aussi 67)

Quoi qu'il en soit, différence et rapprochement s'introduisent donc à nouveau, à la fois successivement et conjointement. La traduction de Blodgett, telle qu'il la décrit, «ne cherche pas avant tout les équivalences. Elle se sert plutôt des images pour les doter d'autres sens. [...] De la sorte, nos images [sont] complémentaires, elles partag[ent] une intimité, tout en étant opposées, encore que réconciliées » (Blodgett 2008: 52) ${ }^{6}$. Dans ses propos comme dans sa pratique, Blodgett fait écho à la «nontraduction» contre "l'idéologie traductionnelle du pareil au même» mise de l'avant par Brault dans Poèmes des quatre côtés (voir Brault 1975: 204) et prône une nonannexion réciproque (voir Blodgett 2008: 48-49).

Si le bilinguisme officiel constitue pour les poètes un acte de violence, leur bilinguisme, lui, se veut mutuel. Sur ce point, la combinaison de formes de traduction métaphorique et pratique, de même que l'alternance des rôles d'auteur et de traducteur entre les poètes, remplissent une fonction essentielle. En effet, les poètes s'en prennent de manière particulièrement systématique à ce qu'Antoine Berman a décrit comme «la condition [...] ancillaire de la traduction, qui répercute sur la condition des traducteurs» (1984: 14). Leur renga translingual se construit précisément contre la hiérarchie habituelle entre original et traduction, entre auteur et traducteur. L'absence de hiérarchie recherchée est d'ailleurs le premier aspect du texte à être présenté en note liminaire:

(9) A renga is a poem for one-legged dancers. As one of us would leap into the air, the leap was made in the faith that the other would complete the leap. Both depend on the other for their pas de deux to be completed. (Blodgett in Blodgett et Brault 1998: 8)

Ainsi chaque texte de départ se présente d'entrée de jeu comme étant incomplet, en attente de l'autre.

Ce que Blodgett ne dit pas à cet endroit mais qui transparaît dans Transfiguration, c'est que le renga, d'après ses spécialistes, est affaire de transformation (voir Konishi 1975: 42; Ogawa 2011: 264). Le nom du recueil renvoie évidemment à cette idée. Le renga est cette traduction qui permet au poème d'aller ailleurs que dans la seule reproduction, et c'est l'un des sens que les auteurs donnent à leur titre (voir Blodgett 2000 : 17). Le renga est aussi transfiguration au sens où il vise au dépassement de soi dans l'échange avec l'autre. Selon Tadashi Ogawa (2001 : 263): «The essential basics of renga lie in both self-abandonment and the participation in $z a$, which is "the opening place" belonging neither to one's self nor to that of the others. » Pour Jin'Ichi Konishi, le processus de création d'un renga « is meant to be pleasant and elevating» (1975: 33). Or, si, comme son nom l'indique et comme sa forme le suggère, Transfiguration met l'accent sur un potentiel de transformation heureuse, cette transformation s'accomplit ici tout en douceur. En ce sens, elle rejoint les valeurs d'harmonie à la base du renga (voir Konishi 1975: 42; Ogawa 2011 : 270-272). La quête d'harmonie fait en sorte que 
la transfiguration soit graduelle et bidirectionnelle. Dans Transfiguration, les lignes de démarcation - entre les poètes, entre leurs langues, entre original et traduction - s'estompent peu à peu dans l'avènement de l'œuvre commune, à laquelle Brault et Blodgett contribuent de manière égale. Selon Sherry Simon:

This is reciprocal writing taken to the furthest point, where words never entirely belong to a single speaker. As the dialogue advances, there is a progressive interconnection between the poets' voices. Transfiguration illustrates the desire to exploit translation's potential to scramble ownership and property. Rather than maintaining separation between languages and identities, these versions shuffle them. The voice of each poet is penetrated by the accent, the vocabulary, the sensibility of the other. (2006:140-141)

Une telle démarche s'oppose au bilinguisme officiel. Mais, tout autant, elle en reprend certains éléments, qu'elle transfigure - au sens cette fois où elle les améliore. Ce n'est donc pas tant la symétrie du bilinguisme officiel qui est mise en cause par Blodgett et Brault, puisque Transfiguration y adhère scrupuleusement. L'opposition est plutôt à une "symétrisation» où la symétrie serait mimée sans qu'il y ait échange véritable. À la symétrie du bilinguisme officiel, Blodgett et Brault ajoutent donc un corollaire qui en change la donne: la transfiguration réciproque.

\section{L'homme invisible/The Invisible Man, ou la double parodie du bilinguisme mortifère}

Le rapport à la symétrie du bilinguisme officiel est plus problématique dans L'homme invisible/The Invisible Man. Il est cette fois pleinement parodique puisque la symétrie se fait ici railleuse: elle est visiblement employée pour être dénoncée - dénonciation qui a été dûment notée par la critique. Ce que la critique a surtout relevé dans cette œuvre copieusement commentée, c'est l'imaginaire de la perte dont elle est imprégnée. Les interprétations disponibles accolent quasi systématiquement le mot «disparition» à L'homme invisible/The Invisible Man et lui donnent d'emblée une signification collective (ainsi, Lasserre 1995-1996: 67; Paré 2007: 1988; Lagacé 1999: 86; J. Melançon 2008: 6). Le bilinguisme du récit semble être le principal vecteur de ces interprétations collectivistes pessimistes, que l'introduction de Robert Dickson (lui aussi poète franco-ontarien) programmait dès la publication en soulignant «l'àpropos de ce texte vis-à-vis [sic] une certaine condition franco-ontarienne de double dépossession " (in Desbiens 2008 [1981] : 18)7. Mobilisé d'une manière qui peut aisément sembler dénonciatrice, ce bilinguisme exemplifierait les conséquences néfastes, relevées par Brault et Blodgett dans leurs essais, d'une application des arrangements linguistiques symétriques à des milieux diglossiques:

c'était la condamnation sans appel, le rejet ontologique du monde que représentait pour Desbiens la langue maternelle française, déchue, dilapidée, indifférenciée, entachée par le malaise et la honte, paralysée et paralysante jusqu'à la désarticulation des structures de la subjectivité. (Paré 2007 : 91)

Si cet aspect ontologique est plus important pour les commentateurs que les enjeux politiques soulevés par le texte, il n'en découle pas moins directement de tels enjeux. Gaston Tremblay, par exemple, refuse de voir dans L'homme invisible/The Invisible Man un «texte politique», affirmant qu'on y trouve "plutôt l'expression de la douleur organique que vivent les auteurs franco-ontariens» (1996: 206). Cela ne 
l'empêche pas de décrire le sujet du récit à l'aide du vocabulaire précis du bilinguisme officiel: «le bilinguisme et le biculturalisme chez les Franco-Ontariens» (1996: 206). Pour Tremblay, la forme du texte fait écho à cette structure binaire. Décrivant l'«ellipse» constituée par le croisement des textes français et anglais agencés par Desbiens, Tremblay relate: "Au centre, on découvrait l'ampleur de la distance qui sépare les deux solitudes de notre pays, et dans le rétrécissement de la courbe, la problématique des Franco-Ontariens» (1996: 207).

La distance allait être confirmée dans toute son ampleur par la réception de la coédition au Canada anglais, où le texte de Desbiens passerait quasi inaperçu et où, dans la seule recension publiée, les différences entre les deux versions ne seraient pas relevées (voir Aubert, 1982: 30; Leclerc et Nolette 2014: 259). Du côté du Québec, L'homme invisible/The Invisible Man faisait l'effet d'une mise en garde - «symptôme de la faiblesse et de la déperdition personnelle et collective» dont le nationalisme présidant à l'institution d'une littérature nationale québécoise avait cherché à "se couper» en rompant avec son héritage canadien-français (Paré 1994 [1992] : 31). Le lien le plus direct avec le bilinguisme officiel est venu sous la plume de Jacques Godbout, un écrivain québécois qui s'est intéressé au statut du français dans les communautés franco-canadiennes où il est langue minoritaire (Godbout 1976; 1981; 1983). Dans L'homme invisible/The Invisible Man, Godbout lisait la preuve de l'ineptie du bilinguisme officiel. La double dépossession évoquée par Dickson serait celle orchestrée par la loi canadienne: sur la page de gauche, un original français déjà traduit de l'anglais, et dès lors déformé; sur celle de droite, la nécessité de se traduire soi-même vers l'anglais. Surtout existentiel aux yeux des critiques franco-ontariens, le sentiment d'anéantissement qui ressort du texte de Desbiens devient argument politique chez Godbout:

Ceux qui ressentent un pincement de cœur à la chanson de [Gilles] Vigneault sur la Louisiane auront mal jusqu'aux tripes à lire The Invisible Man. Il faudrait que Desbiens offre son livre à la bibliothèque du Parlement d'Ottawa, et qu'on le place à côté de la Constitution canadienne, comme mémento. (1983: 59)

Situer l'argument de Godbout par rapport au texte de Desbiens implique de relever la position spécifique d'où il émane. En effet, si le Québec et l'Ontario francophones partagent une même origine canadienne-française, des différences contextuelles - dont certaines sont issues de la démographie et d'autres, du mouvement de repli territorial ayant présidé à l'avènement d'une nation (et d'une littérature nationale) québécoise(s) (voir Hotte 2000b : 53-54 ; Ladouceur 2010 : 190-191) - les séparent quant à leur rapport au bilinguisme. Au Québec, la menace que l'anglais dominant représente a pu être contenue par un pacte imposant le français comme langue véhiculaire. Ce pacte est politique et juridique, comme en témoigne l'adoption de la Charte de la langue française en 1977. Il est également littéraire, la littérature québécoise ayant dès les années 1960 fait de l'emploi du français comme langue principale de ses textes le symbole du statut véhiculaire qu'il s'agissait de lui donner à l'échelle de la société (voir Leclerc 2010: 189). Dans un tel contexte, le bilinguisme littéraire est difficilement acceptable (voir Grutman 2000: 144-145; Godbout 1972: 153) tant il apparaît comme un retour en arrière. Reste que le pacte québécois d'unilinguisme français n'est évidemment pas accessible aux francophones des autres provinces canadiennes, puisqu'ils sont en situation minoritaire. Qu'ils le perçoivent ou non comme une menace, le bilinguisme est pour eux une composante, voire une condition 
de leur identité francophone (voir Poplack 1988; Ladouceur 2010 : 193). Pas étonnant que l'homme invisible ait deux langues maternelles sur la page française et une seule - l'anglais - sur l'anglaise (voir Desbiens 2008 [1981] : 39).

Le mémento auquel Godbout fait allusion, rappelons-le, est par définition non seulement un aide-mémoire, mais aussi une prière pour les défunts. Faire de L'homme invisible/The Invisible Man un mémento, c'est en offrir une lecture où la projection idéologique a préséance sur la description. Godbout se réfère d'ailleurs au texte de Desbiens en omettant le versant français de son titre, puisqu'à ses yeux l'ouvrage relate de toute façon le glissement vers l'anglais que le bilinguisme officiel canadien entérinerait. La perspicacité du lien qu'il propose avec le bilinguisme officiel n'est cependant pas en cause. Dès l'incipit, en effet, la présentation du protagoniste joint à une mise en scène parfaite de la symétrie du bilinguisme officiel la révélation d'un rapport inégalitaire entre les groupes linguistiques:

(10)

L'homme invisible est né à Timmins, Ontario.

Il est Franco-Ontarien.
The invisible man was born in Timmins, Ontario.

He is French-Canadian.

(Desbiens 2008 [1981]: 1) $)^{8}$

Selon la plupart des critiques, l'appellation «French-Canadian» de la page anglaise, qui amalgame tous les francophones canadiens, dérobe son identité culturelle propre au personnage (voir Lasserre 1995-1996: 66; Killeen 1997: 66; Hotte 2000a: 168). En traduisant «Franco-Ontarien» par «French-Canadian», Desbiens montre qu'il maîtrise les usages culturels des deux univers linguistiques qu'il juxtapose. En termes traductologiques, on pourrait dire qu'il opte pour une équivalence fonctionnelle (voir Leclerc et Nolette 2014: 269). En effet, s'il a une existence historique avérée, le terme de Canadien français est tombé en désuétude dans les diverses communautés franco-canadiennes après la nationalisation de la culture québécoise dans les années 1960 (voir Hotte 2000b: 53-54). C'est dire que le texte anglais de Desbiens adopte un terme qui, en français, serait anachronique. Ce faisant, l'auteur met en relief la vision dépassée, simplificatrice et stéréotypée des francophones qui continue d'être véhiculée dans les discours de langue anglaise au Canada. Il évoque à nouveau cette vision plus loin dans le texte, lorsqu'il affirme, encore une fois uniquement sur la page anglaise:

(11) Drunken French-Canadian fiddlers play sad music in the background. (Desbiens 2008 [1981] : 43a)

En insistant sur les éléments folkloriques associés à la culture canadienne-française, la page anglaise situe la culture canadienne-française dans un temps reculé - révélant par ce «denial of coevalness» (Fabian 1983: 31) un rapport colonial entre les deux cultures dites fondatrices du Canada.

Tout le récit de Desbiens fait ressortir l'écart de poids symbolique entre les deux langues qu'il met symétriquement en regard ${ }^{9}$. Ainsi la page française est-elle perméable à l'anglais alors que l'inverse n'est pas vrai. On le voit déjà dans l'incipit où, tandis que l'expression «Franco-Ontarien» ne trouve pas sa place sur la page anglaise, la page française contient un calque de l'anglais: c'est sans préposition que l'homme invisible «est né à Timmins, Ontario». De tels calques sont fréquents sur la page 
française de L'homme invisible/The Invisible Man. Ils apparaissent - tels «fortunément» et «infortunément» (Desbiens 2008: 17f) - même dans des passages qui n'ont pas d'équivalents sur la page anglaise. La traversée des références culturelles suit un schéma similaire. Tandis que de nombreuses références anglo-américaines s’intègrent aisément à la page française, l'émission de télévision québécoise Les Beaux Dimanches a un rayonnement trop restreint pour se rendre jusqu'à la page anglaise. À l'opposé, la présence d'une chanson de Bob Dylan en traduction française sans qu'aucune mention directe en soit faite sur l'une ou l'autre page suggère un lecteur cible assez familier avec cette chanson pour la reconnaître même transformée par l'autre langue:

(12) Comment ça file d'être tout seul comme une meule à Timmins Ontario. (Desbiens 2008 [1981] : 16f)

Certes, les écrivains français Rimbaud et Baudelaire, figures de la littérature mondiale que Desbiens récupère comme personnages, possèdent le statut nécessaire pour voyager vers la version anglaise; mais ils ressortent dégradés de leur passage en Ontario français. Victimes de l'inégalité des univers où ils sont transposés, ils servent moins d'influence qu'ils ne reçoivent celle de la culture anglo-américaine:

(13) Rimbaud est peigné comme Bob Dylan. (Desbiens 2008 [1981] : 15f)

À un Rimbaud rétrogradé fait face Audie Murphy, héros de guerre, vedette de cinéma et idole de l'homme invisible enfant. Audie Murphy «ne parle pas français/doesn't speak French» (Desbiens 2008 [1981] : 6), tandis qu'on peut lire l'anglais de Rimbaud sur les deux pages:

\begin{tabular}{l|l}
{$[\ldots]$} & {$[\ldots]$} \\
"I don't need this shit man!...» & $\begin{array}{l}\text { "I don't need this shit and I don't need } \\
\text { répond Rimbaud en éteignant sa } \\
\text { cigarette dans le sourire vitreux d'un } \\
\text { cendrier du bien-être social. }\end{array}$ \\
"There must be a way out of here. I'm \\
heading south!"
\end{tabular}

(Desbiens 2008 [1981] : 15)

Telle que la manie le poète, la formule de l'édition bilingue est bel et bien subvertie d'une manière qui met le bilinguisme officiel en accusation. De la traduction de l'anglais vers le français des documents gouvernementaux, Desbiens renverse la dynamique en plaçant son français sur la page que les codes de l'édition bilingue assignent à la langue source. Toutefois, par le jeu des influences inégales d'une langue à l'autre, il opère aussitôt un nouveau renversement, qui suggère la difficulté qu'a le français ontarien à occuper cette position. En somme, Desbiens se sert de la symétrie des pages mises en regard pour dénoncer la fragilité d'une langue source incapable d'agir comme telle tant la langue cible est envahissante. On a ici affaire à un récit d'illégitimité radicale, où le poète se forge paradoxalement, à même le texte de la Loi sur les langues officielles, une position de hors-la-loi - invisible parce que hors la langue telle qu'officiellement désignée, et dès lors hors la représentation ${ }^{10}$ : 
(15)

Il feuillette le billet aller-retour de sa langue.

Le billet prend feu et se recroqueville dans le cendrier de sa bouche.
[...]

His pain has no name.

(Desbiens 2008 [1981] : 43)

Godbout relève avec justesse la douleur occasionnée par l'anglais dominant dans le récit. Par contre, il fait silence sur le versant français de cette douleur, sur la domination exercée par le Québec francophone. Pourtant, le passage qu'on vient de lire est la conclusion du séjour de l'homme invisible au Québec. Et la douleur qui y est exprimée est explicitement liée à la déception que lui occasionne ce séjour dans un lieu où il n'est pas arrivé à se reconnaître:

(16) He thought all French-Canadians worthy of their name belonged there. Sunken ships in his sunken eyes. (Desbiens 2008 [1981] : 43a)

Certes, comme en témoigne l'incipit, c'est bien sur la page anglaise que l'homme invisible disparaît le plus complètement. Toutefois, la page française n'a pas pour lui une fonction de refuge. Elle ne le préserve pas de l'invisibilité. S'il peut être nommé sur la page française, son statut de Franco-Ontarien n'est pas toujours perçu par les Québécois qu'il rencontre. Lorsqu'il l'est, il est source d'exotisme. Après avoir quitté son Timmins natal puis passé du temps à Toronto, l'homme invisible aboutit au Québec, où il «falls in love in French» (Desbiens 2008 [1981] : 26a). Or, sa première compagne québécoise, malgré la vaste expérience des rencontres qui lui est supposée, est sans repères devant lui:

(17) Il y a une rumeur qui dit qu'elle couche avec tout ce qui bouge. Mais elle n’a jamais couché avec un homme invisible. (Desbiens 2008 [1981] : 22f)

Dans son ignorance, ce personnage du nom de Pauline n'est pas si différent de Blodgett, dont la dénonciation du bilinguisme officiel réduisait celui-ci à une relation «Canadian-Québécois» (1982: 32). Il n'est pas si différent de Godbout, qui reconnaît les autres francophones du Canada, mais au passé seulement. En prenant parti pour le français contre l'anglais, Godbout ne sort pas des catégories établies par l'État, qui ne tiennent pas compte de "l'espace tiers» (Bhabha 1994; 1996) existant entre les entités qu'elles délimitent ${ }^{11}$. L'homme invisible/The Invisible Man, de son côté, met en scène un brouillage entre les catégories prédéfinies:

(18) He doesn't know if he's going to survive the war.

He doesn't even know whose side he is supposed to be on. (Desbiens 2008 [1981]: 34a)

L'illégitimité mise en scène par Desbiens est donc plus polysémique que ne l'envisage Godbout.

Dans cette perspective, le rôle d'une figure comme celle d'Audie Murphy ne tient pas seulement à son ignorance du français. Le segment où elle intervient laisse entrevoir un entrelacement plus complexe de significations: 


\section{(19)}

Tout le monde sait que les cow-boys ne parlent pas français.

Audie Murphy ne parle pas français. L'homme invisible est Audie Murphy. Il sait comment mourir.

«Hey, you sure know how to die!...» lui dit un de ses amis.

L'homme invisible, immédiatement

flatté, se fait tirer et meurt souvent.
Everyone knows that cowboys don't speak French.

Audie Murphy doesn't speak

French. The invisible man is Audie Murphy. He knows how to die.

"Hey, you sure know how to die!..." says one of his friends.

The invisible man, immediately flattered, gets shot a lot and dies as often as possible.

(Desbiens 2008 [1981]: 6)

Lucie Hotte est la seule critique à avoir porté une attention méticuleuse à la figure d'Audie Murphy. Son analyse fait voir le contraste entre l'homme invisible, qui «sait comment mourir», et Audie Murphy, dont la notoriété tient précisément à ce qu'il «ne savait pas mourir», à "son “aptitude" à survivre à tout» (Hotte 2000a: 167). Elle en conclut à un échec de l'identification de l'homme invisible à Audie Murphy. De fait, l'emploi de l'anglais pour décrire la mort du protagoniste sur les deux pages souligne l'enjeu linguistique de cette mort et en offre une performance efficace. Sauf que la mort de l'homme invisible, constamment recommencée, est justement une performance. Elle contredit les promesses du bilinguisme officiel, mais elle parodie aussi - la répétition d'un événement définitif démentant nécessairement celui-ci - les affirmations nécrologiques émanant du Québec à l'endroit des communautés francophones des autres provinces ${ }^{12}$. C'est dire qu'en plus d'offrir une parodie du bilinguisme officiel, Desbiens offre une parodie... de cette parodie.

\section{Brouillages polysémiques}

La réécriture du bilinguisme officiel à laquelle procède Transfiguration transforme la traduction et le bilinguisme en actes bienveillants, en rapprochement choisi. En revanche, dans L'homme invisible/The Invisible Man, la proximité est à la fois inévitable et insoutenable. C'est là une distinction dont Sherry Simon souligne les enjeux: [When] translation is [...] a benevolent act of generosity towards a friend from distant lands, transactions across languages do not threaten the integrity of either host or receiving culture. But when two languages mingle relentlessly, [...] translation is put to the test» (2006: 9). Les deux textes exemplifient le second cas mentionné par Simon, mais ils ne mettent pas la traduction à l'épreuve au même degré. Blodgett et Brault mêlent leurs voix, voire les fusionnent à l'occasion d'un acte poétique; mais ils continuent de bénéficier à la fois de la confiance que procure l'amitié et de la distance, entre l'Alberta et le Québec, de leurs contrées somme toute éloignées. Pour Desbiens, il n'y a pas de lieu sûr où retourner hors de la rencontre des langues. Là où la transfiguration, d'après les différentes acceptions du terme, produit une amélioration, un embellissement glorieux, le protagoniste de L'homme invisible/The Invisible Man, lui, s'exprime dans un langage "prolétarisé» (Lasserre 1996: 38) et est menacé d'effondrement. À la transfiguration des premiers répond l'altération du second, cette déviation néfaste qui le mène à l'exclusion. Le contraste entre les deux types de rapport à la traduction est frappant. Il ne s'agit pas pour autant d'en tirer une nouvelle 
dualité à l'intérieur de la dualité linguistique canadienne, dans une zone frontière dont on a vu qu'elle s'avère elle-même éminemment différenciée. Plutôt, lire ces textes à la lumière l'un de l'autre permet d'interroger, en même temps que le contraste, la perméabilité de leur lien avec le bilinguisme officiel.

Récit utopique, Transfiguration reste ancré dans l'histoire de la minorisation franco-canadienne qui se trouve au cour de L'homme invisible/The Invisible Man. Cette histoire, soumise au thème de la nature, n'y fait d'apparitions que discrètes; ces apparitions n'en sont pas moins éloquentes. Par exemple, c'est à Brault qu'est due, dès son premier poème, l'introduction d'oiseaux dans le recueil (voir Blodgett 2000 : 17). Le premier oiseau qu'il retient, celui qui lui permet de faire entendre sa voix, est l'alouette (voir Brault in Blodgett et Brault 1998: 11). Ce choix ne saurait être innocent: faut-il rappeler la charge symbolique associée à l'alouette en contexte québécois et canadien? Chez les enfants canadiens-anglais, l'oiseau qu'on plume de la chanson traditionnelle constitue souvent l'une des premières évocations du Canada français. De cette même chanson, Félix Leclerc s'est inspiré pour écrire "L'alouette en colère» durant la crise d'Octobre. Il y dénonçait le sort fait à son "fils dépouillé», dont «on ne reconnaît pas» la langue maternelle (1972). On ne s'étonnera pas, dans ces circonstances, que l'alouette de Brault apparaisse dans un climat où, malgré l'extase qui conclut la strophe, pointe d'abord la dysphorie:

(20) au vent d'été les soleils traqués d'ouest en est confient leur semence les jardins sous la sécheresse brûlent avec l'alouette perchée sur son extase (Brault in Blodgett et Brault 1998: 11)

Empreinte des connotations négatives de son histoire, l'alouette brûle du fait de la sécheresse de son environnement.

Ces connotations négatives, Blodgett - dont les images sont généralement moins sombres que celles de Brault (voir Blodgett 2007: 53) - les atténue d'abord. Maintenant le texte dans le registre de la nature, il retient plutôt de l'oiseau son vol. Dans sa traduction, il évoque l'élévation de l'alouette par son choix de prépositions:

(21) under the summer wind suns

harried from west to east

bestow their seed

gardens in drought

burn as the lark burns

high upon its ecstasy (Brault tr. Blodgett in Blodgett et Brault 1998: 11)

Dans le trio des prépositions «au», «sous» et «sur» retenues par Brault, il introduit une progression graduelle: «under», «in», «upon $»^{13}$. Dans le poème suivant, qui constitue sa réponse à celui de Brault sur l'alouette, l'élévation de l'oiseau devient explicite:

(22) bright seminarian devotee

of summer and the sun the lark

ascending rises in the air

beyond the reach of joy (Blodgett in Blodgett et Brault 1998: 12, je souligne) 
Reprenant à Brault le lien entre alouette et extase, Blodgett entraîne l'oiseau plus loin encore dans la joie.

La conclusion de son poème montre pourtant que Blodgett n'est pas sourd au symbolisme de l'oiseau - et même qu'il en joue, lui juxtaposant un autre symbole politique canadien :

(23) voice suspended

in the pitch

of solitudes (Blodgett in Blodgett et Brault 1998: 12, je souligne)

Au pluriel, «solitudes» évoque les deux solitudes rendues célèbres par le romancier Hugh MacLennan (1945), comme si Blodgett avait répondu au cliché de Brault (l'alouette) par un autre cliché (les deux solitudes). Les moments de rencontre «où le son est le sens", où l'anglais et le français se fondent, ne durent que le temps du "fi-bi»" d'une mésange à capuchon noir (Brault in Blodgett et Brault 1998: 41), et les auteurs sont vite rattrapés par le discours social à l'arrière-plan de leur conversation poétique. La célébration de l'amitié à laquelle ils se livrent n'élude pas la précarité de leur démarche. Lorsque Blodgett demande: "are we agreed on this my friend " in Blodgett et Brault 1998: 40; voir aussi exemple 1), il esquisse un terrain commun avec son correspondant, mais soulève en même temps un doute quant à l'étendue de ce terrain. Tant la forme interrogative que l'isolement du «my friend» à la fin du vers transmettent ce doute. La traduction de Brault, qui place l'"ami» au centre du vers, accentue visuellement l'espace qui sépare les correspondants:

(24) sommes-nous mon ami d'accord sur ceci (Brault in Blodgett et Brault 1998: 40)

Citée plus haut (voir exemple 2), la réponse de Brault au poème de Blodgett vaut d'être reprise dans une interprétation légèrement différente, d'où ressortent certaines tensions. Dans le premier vers de cette réponse, Brault, on l'a vu, reprend à son compte l'amitié introduite par Blodgett. Écrivant "faisant amitié avec des mots», il semble décrire la démarche qui est la leur dans Transfiguration. Toutefois, lorsqu'il fait dépendre cette amitié de «mots / où le son est le sens », il restreint d'autant l'étendue de leur terrain commun. La restriction se confirme au troisième vers, qui ramène avec l'intervention d'une mésange les poètes au thème de la nature. Reportant l'amitié sur une mésange, Brault s'en distancie. Blodgett le signale dans son commentaire sur son activité traductionnelle dans Transfiguration: ce passage est «one of the rare moments in the book in which the character of our relationship was raised " (2000: 20). Brault le contient en y mettant fin.

Dans un mouvement inverse, l'enrichissement que se promettent Blodgett et Brault affleure au travers des enjeux diglossiques de L'homme invisible/The Invisible Man. Ici aussi, le texte avalise des interprétations divergentes. Dans le passage qui suit, quand la page française reprend en le déformant un vers célèbre de Verlaine, la page anglaise omet d'y faire référence: 
Les saisons se pilent les unes par-dessus les autres à une vitesse folle.

Le temps passe si vite que les jours sont devenus un clignotement continu.

Les violons saoulons de l'automne jouent une musique triste dans les yeux de l'homme invisible.

(n)

\section{$[\ldots]$}

(n) 
[...]

Son autobus part dans une vingtaine de minutes.

Au-dessus de la gare le ciel est bleu et beau. Comme tout ce qui est beau, il ne répond à aucune question.
As the bus pulls out of the station, the invisible man looks out the window.

The sky is lightning blue and beautiful.

Like everything else that is beautiful, it answers no questions.

(Desbiens 2008 [1981] : 46)

\section{Les interstices grouillants du bilinguisme officiel}

En guise de conclusion, j'aimerais offrir deux remarques. La première concerne la relation trouble, à la fois idéologique et formelle, que les textes de Desbiens d'un côté et de Brault et Blodgett de l'autre entretiennent avec le bilinguisme officiel canadien et avec les pratiques traductionnelles qui lui sont associées. Cette remarque veut mettre l'accent sur la similitude de leurs approches. Que ces textes se montrent tous deux critiques à l'endroit du bilinguisme officiel semble aller de soi, surtout si on prend en compte le discours social en circulation dans les milieux où frayent les auteurs et le fait qu'ils ont contribué à ce discours. Ce qui frappe davantage dans ce contexte dénonciateur, c'est leur proximité avec le modèle du bilinguisme officiel. Ici, la dénonciation n'est pas une dissociation, puisqu'à travers elle le bilinguisme officiel est encore pris pour cadre. Plus encore, il devient un puissant moteur d'écriture. Les univers mis en place dans Transfiguration et dans L'homme invisible/The Invisible Man se construisent précisément à partir de ce fondement de l'imaginaire national canadien, à partir des politiques officielles sur le bilinguisme.

Plusieurs textes littéraires canadiens thématisent le bilinguisme anglais-français dans ses liens avec le discours de l'État. On n'a qu'à penser à Fall on Your Knees d'Ann-Marie MacDonald, qui a connu un succès spectaculaire. L'écrivaine y recadre le plurilinguisme de ses personnages immigrants à l'aune du bilinguisme officiel. En témoignent les petits-enfants de la famille Luvovitz, dont la première génération était locutrice du yiddish et la seconde avait passé à l'anglais: "They speak French at home, English at school and Yiddish with every second shopkeeper. Real Canadians » (1997: 559). Douglas Coupland, un autre écrivain à succès, aborde directement la Loi sur les langues officielles dans son Souvenir of Canada: "sometimes the French name is so bizarre and cool looking", écrit-il à propos des étiquettes bilingues des produits, «that you just have to accept the fact that Canada is, in some obtusely Star Trek manner, a parallel-universe country, with two variations existing alongside each other; and through the miracle of nationhood, we bounce back and forth between the two universes» (2002: 10). Ce qui distingue Transfiguration et L'homme invisible/The Invisible Man de tentatives de ce genre est le travail sur la forme même du bilinguisme officiel, c'est-à-dire sa forme traductionnelle. Le discours sur, dans leur cas, est aussi un discours par. Dans son heureux redoublement du bilinguisme officiel, l'édition bilingue donne forme au récit de Desbiens, ce qui permet de le faire advenir à la représentation. Sans compter que L'homme invisible/The Invisibile Man est le texte qui a donné à la littérature franco-ontarienne cette existence légitimée dont le personnage souffre d'être dépourvu! Chez Brault et Blodgett, le contexte de la loi réapparaît - véritable 
retour du refoulé -, malgré la démarche consciente que les poètes entreprennent pour s'y soustraire.

La seconde remarque est méthodologique et vise à prendre en compte les divergences existant entre deux textes qui font pourtant sensiblement le même exercice. Transfiguration et L'homme invisible/The Invisible Man se situent tous deux à la croisée des langues officielles et font tous deux ressortir cet interstice - ou espace tiers - dont ils émanent. En même temps, à l'intérieur de cet interstice, les deux textes occupent des positions radicalement différentes. En ce sens, leur lecture conjointe invite à un comparatisme ne portant exclusivement ni sur les imaginaires respectifs de l'anglais et du français au Canada ni même sur une zone frontière entre les deux qu'on imaginerait unifiée, ou uniformément perturbatrice. Elle invite à un comparatisme attentif aux divergences existant entre diverses modalités de la rencontre des langues, même quand ces modalités semblent rapprochées. Elle invite à une analyse de la positionnalité qui n'exclut pas les recoupements, mais ne s'y résume pas non plus. La prise en compte de différentes pratiques littéraires traductionnelles illustrant différents rapports à la traduction, à l'intérieur du cadre que fournit le bilinguisme officiel canadien, me semble constituer un premier pas en ce sens ${ }^{15}$.

\section{REMERCIEMENTS}

Nicole Nolette, durant ses études doctorales sous ma supervision, a rassemblé certaines ressources bibliographiques pour la préparation du présent article. Ses réflexions des dernières années sur la traduction ludique m'ont permis d'approfondir ma lecture de L'homme invisible/ The Invisible Man. Lorsqu'il était étudiant à la maîtrise avec moi, Mathieu Simard a produit une fine lecture de Transfiguration qui a attiré mon attention sur certains détails pertinents pour cet article. Qu'ils en soient remerciés.

\section{NOTES}

1. Selon les données de Statistique Canada, «les minorités de langue officielle (francophones à l'extérieur du Québec et anglophones au Québec) sont plus bilingues que les majorités. [...] Parmi les minorités de langue officielle, les anglophones du Québec affichaient un taux de bilinguisme de $61 \%$ (comparativement à $6 \%$ chez les anglophones dans le reste du Canada), alors que les francophones en dehors du Québec avaient un taux de bilinguisme de $87 \%$ (comparativement à $38 \%$ chez les francophones du Québec)» (Lepage et Corbeil 2013: 4).

2. CCN (16 novembre 1999). Pause-lecture avec la Gouverneur générale [sic] : le Conseil des Arts du Canada annonce les noms des lauréats des Prix littéraires du Gouverneur général de 1999. Fil de presse.

3. Sans faire de lien avec le renga, Charlotte Melançon recense "vingt-sept oiseaux différents» dans Transfiguration et qualifie l'ouvrage de «bref traité d'ornithologie» (2000: 12).

4. Simon (1994: 64-65) cite elle aussi ces passages de l'essai de Brault.

5. Reproduction autorisée par les Éditions du Noroît.

6. Blodgett traite ici de sa traduction d'un autre recueil de Brault, Au fond du jardin.

7. Dickson soulignait cet à-propos avec dérision, affirmant d'abord qu'il serait «lourd, pédant et gauche (not necessarily in that order) de [le] souligner» (in Desbiens 2008 [1981] : 18). Leclerc (2010: 310-314) analyse cette interprétation relativement au concept de bilinguisme soustractif que les chercheurs travaillant sur les minorités francophones du Canada ont emprunté à la psycholinguistique.

8. Cette pagination est celle qui fait partie du texte lui-même plutôt que le folio de l'édition de 2008. C'est elle qui sera utilisée dans tout l'article. Dorénavant, les citations d'un seul des côtés de la page double seront suivies de la lettre $a$ pour anglais et $f$ pour français. La reproduction est autorisée par les Éditions Prise de parole.

9. Dans Leclerc 2010: 307 et Leclerc et Nolette 2014: 270, je fais également ressortir cet écart, en convoquant certains des mêmes exemples. 
10. Selon Marie-Chantal Killeen, «le récit fait état de la difficulté [...] irréductible de faire entrer la langue des Franco-Ontariens dans le jeu de la représentation» (1997: 181). Voir Leclerc 2010: 325, n. 98; et Leclerc et Nolette 2014: 271.

11. Homi Bhabha (1996: 54) présente cet espace comme «the contaminated yet connective tissue between cultures - at once the impossibility of culture's connectedness and the boundary between", qui «introduce[s] into the polarization of liberals and liberationists the sense that the translation of cultures is a complex act [...] that generates borderline affects and identifications [...]». Certes, les minorités postcoloniales que Bhabha situe dans l'espace tiers sont rarement, tel l'homme invisible, des sujets caucasiens établis de longue date dans un territoire. Néanmoins, puisque Bhabha l'envisage comme un espace de traduction, le concept peut être mis en lien avec les littératures des minorités linguistiques au Canada (voir Simon 1999). Citant Walter Benjamin, Bhabha y voit en effet «that element in a translation which does not lend itself to translation» (voir Bhabha 1994: 224; Benjamin 1968: 75). On ne saurait mieux, me semble-t-il, décrire l'écriture de L'homme invisible/The Invisible Man. Pour une critique de la hiérarchisation des langues que maintient le modèle de Bhabha, voir Simon 1995 et Leclerc 2010: 82-90.

12. De mémoire récente, la plus célèbre est celle de l'écrivain québécois Yves Beauchemin, qui, en 1991, avait qualifié les francophones du Canada vivant hors Québec de «cadavres encore chauds du fédéralisme canadien». Le mémento de Godbout appartient certes à ce type de discours, que Godbout a tenu suffisamment fréquemment pour faire l'objet d'une caricature dans le roman autobiographique Moncton mantra de l'écrivain acadien Gérald Leblanc (1997: 106).

13. Je dois cette observation à Mathieu Simard.

14. Ce clignotement évoqué par Desbiens, Paré (1994: 20-21) s'en sert pour décrire une dialectique d'apparitions et de disparitions identitaires qui serait propre à la culture et à la littérature francoontariennes. Cette interprétation, on le voit, n'épuise cependant pas le fonctionnement du clignotement dans le texte. Pour d'autres exemples de clignotement plus ludique, voir Leclerc et Nolette 2014: 272-273.

15. Outre les textes étudiés ici, Two shores/Deux rives de Thuong Vuong-Riddick (1995) est une autre édition bilingue digne d'intérêt de ce point de vue. L'auteure d'origine vietnamienne et établie en Colombie-Britannique présente un recueil de poèmes autobiographiques qu'elle formule dans les deux langues officielles. Les divergences entre les versions, la traversée des langues entre la page de gauche et celle de droite, de même que le bouleversement des attentes quant aux langues de départ et d'arrivée n'en sont pas exclus.

\section{RÉFÉRENCES}

Aubert, Rosemary (avril 1982): New Stars in the Galaxy of Canadian Poetry. Quill \& Quire. 30. Aunger, Edmund A. (Semaine du $1^{\text {er }}$ au 7 mars 2012): Mettez fin au bilinguisme et épargnez des milliards de dollars? Le Franco (Edmonton). Consulté le 30 juin 2013. www.ualberta. $\mathrm{ca} / \sim$ eaunger/discours/Opinion-2012.pdf

Bélanger, Louis (2000): Patrice Desbiens: au cœur des fictions sociales. In: Hédi Bouraou I (dir.), La littérature franco-ontarienne. État des lieux. Sudbury (Ont.): Série monographique en sciences humaines, Université Laurentienne, 197-226.

Benjamin, Walter (1968): Illuminations. (Traduit par Harry Zohn.) New York: Shocken Books. Berman, Antoine (1984): L'épreuve de l'étranger. Traduction et culture dans l'Allemagne romantique (Herder, Goether, Schlegel, Novalis, Humboldt, Schleiermacher, Hölderlin). Coll. «NRF». Paris: Gallimard.

Blodgett, E. D. (1982): Configuration. Essays in the Canadian Literatures. Downsview: ECW Press.

Blodgett, E. D. (2000): Transfiguring Transfiguration. ellipse. 64: 16-23.

Blodgett, E. D. (2008): L'innombrable Brault. In: François Hébert et Nathalie Watteyne (dir.), Précarités de Brault. Coll. «Convergences». Québec: Nota bene, 47-55.

Blodgett, E. D. et Brault, Jacques (1998): Transfiguration. Coll. «Résonance». Saint-Hippolyte (Québec) et Toronto: Noroît et BuschekBooks.

Brault, Jacques (1989): Sur la traduction de la poésie [1977]. In: La poussière du chemin. Montréal: Boréal, 1989. 
CôTÉ, Nicole (2007): The Braultian path to the Other: Estrangement and nontranslation. In: Silke Horstкотte et Esther Peeren (dir.), The Shock of the Other: Situating Alterities. Collection Thamyris/Intersecting. Amsterdam: Rodopi, 161-170.

Coupland, Douglas (2002): Souvenir of Canada. Vancouver: Douglas \& McIntyre.

Desbiens, Patrice (2008 [1981]): L'homme invisible/The Invisible Man. In: L'homme invisible/ The Invisible Man suivi de Les cascadeurs de l'amour. Coll. «Récits». Sudbury (Ont.): Prise de parole [Sudbury et Moonbeam: Prise de parole et Penumbra Press].

Fabian, Johannes (1983): Time and the Other: How Anthropology Makes Its Object. New York: Columbia University Press.

Godbout, Jacques (1972): D’amour, P.Q. Montréal et Paris: Hurtubise HMH et Seuil.

Godbout, Jacques (1976): Avant-propos. In: Roger Auger, Je m’en vais à Régina. Montréal: Leméac, ix-xi.

Godbout, Jacques (1981): Les têtes à Papineau. Paris: Seuil.

Godbout, Jacques (1983) : Mardi 20 avril. In: Journal d'hiver (décembre 1981-avril 1982). Liberté 25(4[148]):59-60.

Grutman, Rainier (2005): La textualisation de la diglossie dans les littératures francophones. In: Jean Morency et al. (dir.), Des cultures en contact. Visions de l'Amérique du Nord francophone. Québec: Nota bene.

Нотте, Lucie (2000a): Entre l'Être et le Paraître: conscience identitaire et altérité dans les œuvres de Patrice Desbiens et de Daniel Poliquin. In: Yvan G. Lepage et Robert Major (dir.), Croire à l'écriture. Orléans (Ont.): Les Éditions David, 163-178.

Hотте, Lucie (2000b): Littérature et conscience identitaire: l'héritage de CANO. In: Andrée ForTin (dir.), Produire la culture, produire l'identité? Coll. «Culture française d'Amérique». Sainte-Foy: Presses de l'Université Laval, 53-68.

Hutcheon, Linda et Marion Richmond (dir.) (1990): Other Solitudes: Canadian Multicultural Fictions. Préface de Marion Richmond, introduction de Linda Hutcheon. Toronto: Oxford University Press.

Juhel, Denis (1999): Proxilité et qualité des traductions. Meta. 44(2):238-249.

Konishi, Jin'Ichi (automne 1975): The art of renga (traduction et introduction par Karen Brazell et Lewis CooK). Journal of Japanese Studies. 1(2):29-61.

LADOUCEUR, Louise (2010): Unilinguisme, bilinguisme et esthétique interculturelle dans les dramaturgies francophones du Canada. International Journal of Francophone Studies. 13(2):183-200.

LAGACÉ, Francis (1999): Apprivoiser sa langue comme une belle étrangère (la minorité dans la minorité: le cas du poète franco-ontarien Patrice Desbiens). In: Christiane Albert (dir.), Francophonie et identités culturelles. Paris: Karthala, 85-106.

LARose, Karim (2004): La langue de papier. Spéculations linguistiques au Québec. Montréal: Les Presses de l'Université de Montréal.

Lasserre, Élisabeth (hiver 1995-1996): Patrice Desbiens: «Je suis le Franco-Ontarien». Nuit blanche. 62: 64-68.

LASSERre, Éslisabeth (1996): Un poète au seuil de l'écriture. L'exiguïté selon Patrice Desbiens. In: Lucie Нотte et François Ouellet (dir.), La littérature franco-ontarienne. Enjeux esthétiques. Ottawa: Le Nordir, 27-42.

Leblanc, Gérald (1997): Moncton mantra. Moncton: Perce-Neige.

LeCLerC, Félix (1972): L'alouette en colère. L'alouette en colère. Album. Philips 6325022.

LECLERC, Catherine (2010): Des langues en partage? Cohabitation du français et de l'anglais en littérature contemporaine. Montréal: XYZ.

LeClerc, Catherine et Nicole Nollette (2014): Pour ou contre la traduction. L'homme invisible/ The Invisible Man de Patrice Desbiens. In: Arnaud Bernadet et Philippe Payen De LA Garanderie (dir.)s Traduire-écrire. De l'expérience à la théorie. Coll. «Signes». Lyon: ENS Éditions, 257-275.

LePAgE, Jean-François et CorbeiL, Jean-Pierre (mai 2013) : L'évolution du bilinguisme anglaisfrançais au Canada de 1961 à 2011. Coll. «Regards sur la société canadienne». Ottawa: 
Statistique Canada. Consulté le 30 juin 2013. <http://publications.gc.ca/collections/collection _2013/statcan/75-006-x/75-006-2013001-4-fra.pdf>

MacDonald, Ann-Marie (1997 [1996]): Fall on your Knees. Toronto: Vintage Canada [Alfred A. Knopf Canada].

MacLennan, Hugh (1945): Two Solitudes. New York: Duell, Sloane and Pearce.

Melançon, Charlotte (2000): Traduire la poésie. Présentation. ellipse. 64: 9-12.

Melançon, Johanne (2008): Relire L'homme invisible/The Invisible Man et Les cascadeurs de l'amour. In: Patrice Desbiens. L'homme invisible/The Invisible Man suivi de Les cascadeurs de l'amour. Sudbury: Prise de parole, 5-15.

Ogawa, Tadashi (2011): A short study of Japanese renga: The trans-subjective creation of poetic atmosphere. In: Anna-Teresa Tymienieck a (dir.), Destiny, the Inward Quest, Temporality and Life. Dossier dans Analecta Husserliana. 109: 257-274.

Paré, François (1994 [1992]): Les littératures de l'exiguïté. $2^{e}$ éd. Ottawa: Le Nordir.

PARÉ, François (1994 ): Théories de la fragilité. Ottawa: Le Nordir.

Paré, François (2007): Le fantasme d'Escanaba. Québec: Nota bene.

Paz, Octavio, Roubaud, Jacques, Sanguineti, Edoardo et Tomlinson, Charles (1971): Renga. Paris: Gallimard.

Poplack, Shanna (1988): Language Status and Language Accommodation Along a Linguistic Border. In: Peter H. Lowenberg (dir.), Language Spread and Language Policy: Issues, Implications and Case Studies. Washington (D. C.): Georgetown University Press, 90-118.

ReID, Scott (1993): Lament for a Notion: The Life and Death of Canada's Bilingual Dream. Vancouver: Whitecap Books.

Simon, Sherry (1994): Le trafic des langues. Traduction et culture dans la littérature québécoise. Montréal: Boréal.

Simon, Sherry (1999): Translating and interlingual creation in the contact zone: border writing in Quebec. In: Susan Bassnett et Harish Trivedi (dir.), Post-colonial Translation: Theory and Practice. Londres \& New York: Routledge.

Simon, Sherry (1995): La culture transnationale en question. Visée de la traduction chez Homi Bhabha et Gayatri Spivak. Études françaises. 31(3): 43-57.

Simon, Sherry (1999): Hybridité culturelle. Montréal: L'Île de la tortue.

Simon, Sherry (2000): The Paris arcades, the Ponte Vecchio and the comma of translation. Meta. 45(1):73-79.

Simon, Sherry (2002): Crossing town: Montreal in translation. Profession. 15-24.

Simon, Sherry (2006): Translating Montreal: Episodes in the Life of a Divided City. Montréal \& Kingston: McGill-Queen's University Press.

TARAVELla, AnneMarie (3 octobre 2011): Rapport sommaire et préliminaire sur les résultats de l'enquête menée auprès des utilisateurs de technologies langagières en avril-mai 2011. Gatineau (Québec): Centre de recherche en technologies langagières (CRTL).

Tremblay, Gaston (1996): Prendre la parole: le journal de bord du Grand CANO. Ottawa: Éditions Le Nordir.

Vaillancourt, François et Coche, Olivier (mai 2009): Official Language Policies at the Federal Level in Canada: Costs and Benefits in 2006. Vancouver: Institut Fraser.

Vaillancourt, François, Coche, Olivier et al. (janvier 2012): Official Language Policies of the Canadian Provinces: Costs and Benefits in 2006. Vancouver: Institut Fraser.

Vuong-Riddick, Thuong (1995): Two Shores/Deux rives. Vancouver: Ronsdale Press.

ZaBus, Chantal (1991): The African Palimpsest: Indigenization of Language in the West African Europhone Novel. Amsterdam et Atlanta: Rodopi. 


\section{ANNEXES}

Annexe 1

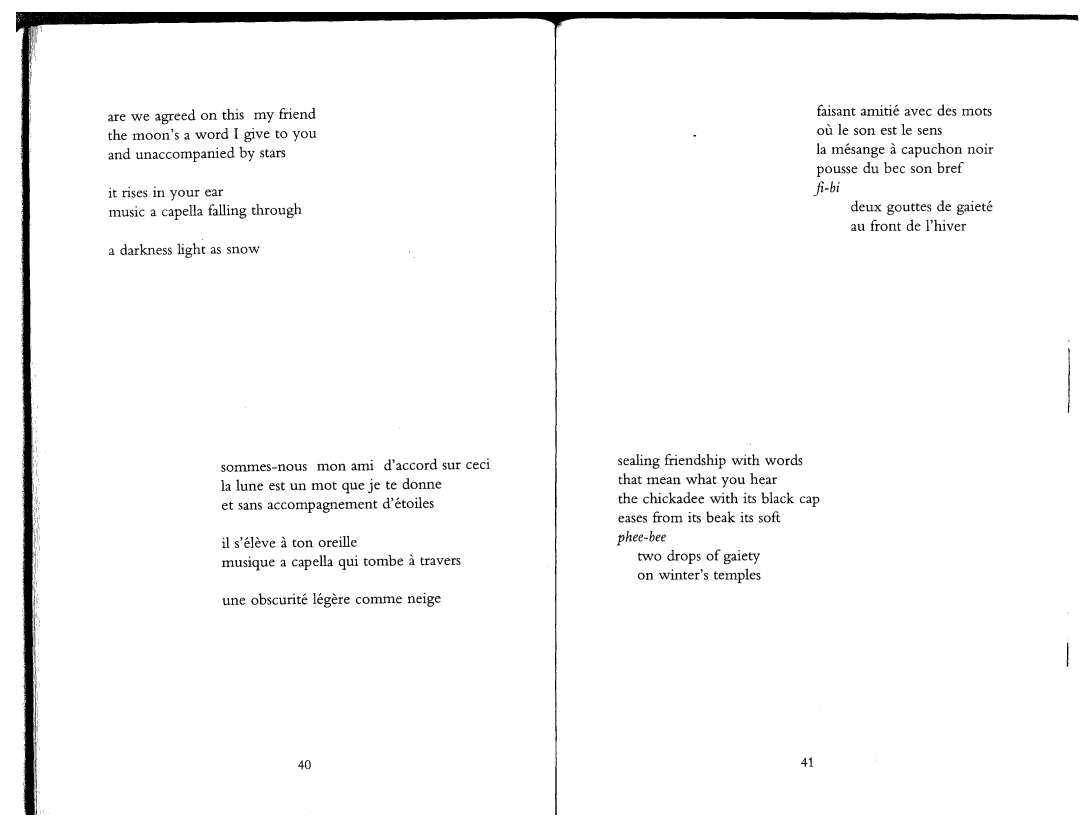

Reproduction autorisée par les Éditions du Noroît. E. D. Blodgett et Jacques Brault (1998). Transfiguration. Coll. «Résonance». Saint-Hippolyte et Toronto: Noroît et BuschekBooks, p. 40-41.

Annexe 2

42.

Les saisons se pilent les unes par-dessus les autres à une vitess

folle.

Le temps passe si vite que les jours sont devenus un clignotement continu.

Les violons saoulons de l'automne jouent une musique triste dans les yeux de l'homme invisible.
Voices in the sky.

Lights in the sky.

Silence cracks like cellophane.

Well well.

Voices from the sky.

The invisible man doesn't quite understand what they're talking about.

He runs out,

goes to a bar and stays until closing time.

The waiter escorts him out.

Goodbye. Goodbye.

A choice of deaths.

Outside, it's winter.

It's telephone pole cold

Cars and skidoos drive thru the invisible man like he was made of smoke, like it was such a sensible, logical thing to do. He makes it home and throws himself on the bed like an old overcoat.

Sweet oblique oblivion

Slowly going crazy like the lightbulb on the ceiling.

The days go by faster and faster.

The sun a strobe light in the sky.

Reproduction autorisée par les Éditions Prise de parole. Patrice Desbiens (2008). L'homme invisiblel The Invisible Man suivi de Les cascadeurs de l'amour. Sudbury: Prise de parole, p. 106-107 [42]. 